\title{
Article
}

\section{Approximation of Fixed Points of Multivalued Generalized $(\alpha, \beta)$-Nonexpansive Mappings in an Ordered CAT(0) Space}

\author{
Mujahid Abbas ${ }^{1,2}\left[\right.$, Hira Iqbal ${ }^{3}$, Manuel De la Sen ${ }^{4, *}$ and Khushdil Ahmad ${ }^{1}[$ \\ 1 Department of Mathematics, Government College University, Katchery Road, Lahore 54000, Pakistan; \\ abbas.mujahid@gmail.com (M.A.); khushdilahmad834@gmail.com (K.A.) \\ 2 Department of Medical Research, China Medical University Hospital, China Medical University, \\ Taichung 404, Taiwan \\ 3 Department of Sciences and Humanities, Lahore Campus, National University of Computer and Emerging \\ Sciences, Lahore 54000, Pakistan; hira.iqbal@nu.edu.pk \\ 4 Institute of Research and Development of Processes, University of the Basque Country, 48940 Leioa, Spain \\ * Correspondence: manuel.delasen@ehu.eus
}

check for updates

Citation: Abbas, M.; Iqbal, H.; De la Sen, M.; Ahmad, K.

Approximation of Fixed Points of Multivalued Generalized $(\alpha, \beta)$-Nonexpansive Mappings in an Ordered CAT(0) Space. Mathematics 2021, 9, 1945. https:// doi.org/10.3390/math9161945

Academic Editors: Yeol Je Cho, Sang-Eon Han and Jong Kyu Kim

Received: 7 July 2021

Accepted: 10 August 2021

Published: 15 August 2021

Publisher's Note: MDPI stays neutral with regard to jurisdictional claims in published maps and institutional affiliations.

Copyright: (c) 2021 by the authors. Licensee MDPI, Basel, Switzerland. This article is an open access article distributed under the terms and conditions of the Creative Commons Attribution (CC BY) license (https:/ / creativecommons.org/licenses/by/ $4.0 /)$.

\begin{abstract}
The purpose of this article is to initiate the notion of monotone multivalued generalized $(\alpha, \beta)$-nonexpansive mappings and explore the iterative approximation of the fixed points for the mapping in an ordered CAT(0) space. In particular, we employ the $S$-iteration algorithm in CAT(0) space to prove some convergence results. Moreover, some examples and useful results related to the proposed mapping are provided. Numerical experiments are also provided to illustrate and compare the convergence of the iteration scheme. Finally, an application of the iterative scheme has been presented in finding the solutions of integral differential equation.
\end{abstract}

Keywords: multivalued nonexpansive mapping; fixed point; monotone; CAT(0) space

MSC: 47H09; 47H10

\section{Introduction and Preliminaries}

Let $M$ a nonempty subset of a metric space $Y$. A subset $M$ is said to be proximinal if for each $v \in Y$, there exists an element $k \in M$ such that

$$
d(v, k)=d(v, M)=\inf \{d(v, y): y \in M\}
$$

where $d(v, M)$ is the distance from the point $v$ to the set $M$. Denote the family of nonempty closed bounded subsets of $Y$ by $\Omega(Y)$, the family of nonempty bounded proximinal [1] subsets of $Y$ by $D(Y)$, and the family of nonempty compact subsets of $Y$ by $\kappa(Y)$. Define the Hausdroff distance, $H(. .$.$) , on \Omega(Y)$ by

$$
H(E, F)=\max \left\{\sup _{e \in E} d(e, F), \sup _{f \in F} d(f, E)\right\} .
$$

The mapping $H$ is called Pompeiu-Hausdorff metric induced by $d$.

A multivalued mapping $\mathcal{U}: Y \longrightarrow \Omega(Y)$ is said to have a fixed point if there exists an element $p \in Y$ such that $p \in \mathcal{U} p$. The set $F(\mathcal{U})$ denotes the set of all fixed points of $\mathcal{U}$. This $p \in Y$ is said to be a strict fixed point (or end point of $\mathcal{U}$ ) if $\mathcal{U} p=\{p\}$. Denote the set of strict fixed points (end points) of $\mathcal{U}$ by $S F(\mathcal{U})$. Clearly, $S F(\mathcal{U})$ is contained in $F(\mathcal{U})$. A multivalued mapping $\mathcal{U}: Y \longrightarrow \Omega(Y)$,

(1) is nonexpansive if

$$
H(\mathcal{U} v, \mathcal{U} y) \leq d(v, y), \quad \forall \quad v, y \in Y
$$


(2) is quasi-nonexpansive if $F(\mathcal{U}$ is nonempty and for any $p \in F(\mathcal{U})$

$$
H(\mathcal{U} v, \mathcal{U} p) \leq d(v, p), \quad \forall \quad v \in Y .
$$

A multivalued mapping $\mathcal{U}: Y \longrightarrow \Omega(Y)$ satisfies the Condition $(\mathrm{C})$ if for any $v, y \in Y$

$$
\frac{1}{2} d(v, \mathcal{U} v) \leq d(v, y) \quad \text { implies that } H(\mathcal{U}, \mathcal{U} y) \leq d(v, y)
$$

A geodesic path is a map, $\xi$, joining two points $v$ and $y$ in a metric space $Y$ from a closed interval $[0, l] \subset \mathbb{R}$ to $Y$ such that $\xi(0)=v, \xi(l)=y$ and $d\left(\xi(q), \xi\left(q^{\prime}\right)\right)=\left|q-q^{\prime}\right|$ for all $q, q^{\prime} \in[0, l]$. In particular, $l=d(v, y)$. The image of $\xi$ is called the geodesic or metric segment joining $v$ and $y$. If the image is unique then it is indicated by $[v, y]$. The space $(Y, d)$ is called the geodesic space if any two points of $Y$ are connected by a geodesic whereas $Y$ is known to be uniquely geodesic if for each $v, y \in Y$, there is exactly one metric segment which joins $v$ and $y$ [2]. A subset $M$ of $Y$ containing every geodesic segment joining any two of its points is said to be convex. A geodesic triangle $\triangle(a, b, c)$ in a geodesic metric space $(Y, d)$ consists of three points in $Y$ where $a, b$ and $c$ are the vertices of $\triangle$ and a geodesic segments between them are the sides of $\triangle$. A comparison triangle for $\triangle(a, b, c)$ in $(Y, d)$ is a triangle $\bar{\triangle}(a, b, c)=\triangle(\bar{a}, \bar{b}, \bar{c})$ in the Euclidean plane $\mathbb{R}^{2}$ such that $d(a, b)=d_{\mathbb{R}^{2}}(\bar{a}, \bar{b})$, $d(a, c)=d_{\mathbb{R}^{2}}(\bar{a}, \bar{c})$ and $d(b, c)=d_{\mathbb{R}^{2}}(\bar{b}, \bar{c})[3,4]$.

Suppose that $\Delta$ is a geodesic triangle in $E$ and $\bar{\Delta}$ is a comparison triangle for $\Delta$. In a geodesic space, if all geodesic triangles of appropriate size satisfy the following comparison axiom called CAT(0) inequality:

$$
d(u, v) \leq d_{\mathbb{R}^{2}}(\bar{u}, \bar{v}), \quad \text { for all } u, v \in \Delta, \quad \bar{u}, \bar{v} \in \bar{\Delta},
$$

then such a geodesic space is said to be a CAT(0) space.

Thus, a CAT(0) space is a particular metric space which does not possess any linear structure. Complete CAT(0) spaces generalize Hilbert spaces to the nonlinear framework. Some examples of CAT(0) spaces are pre-Hilbert space [5], Hadamard manifold, R-trees [6], hyperbolic metric spaces [7] and Euclidean building [8]. We refer the readers interested in detailed study of such spaces to [2] and references therein.

A study of sufficient conditions for the existence of fixed points of multivalued contraction and nonexpansive mappings employing the Hausdroff metric was first carried out by Markin [9] which was later extended by Nadler [10]. A topological structure which relies on the properties of distance function defined on the domain and induced distance function on a codomain of multivalued contraction mapping plays vital role in proving the existence of fixed points of such mappings. Fixed point theory for multivalued contraction mappings initiated by Nadler [10] has been extended using different generalized distance structures such as $b$-metric, rectangular metric, partial metric, and dual metric [11-14].

Existence of fixed points of single valued and multivalued nonexpansive mappings however requires a rich geometric structure in addition to the topological structure of underlying domain see [15-17] and references therein. Kirk [6] instituted the study of fixed points in CAT(0) spaces. He established the existence of a fixed point for a nonexpansive single-valued mapping on bounded closed convex subset of a complete CAT(0) space. In fact, CAT(0) spaces constitute a suitable framework to obtain fixed points of nonexpansive mappings and its various generalizations [18-20]. Fixed point results of multivalued mappings in CAT(0) spaces have various applications in differential equations, optimization, control theory, graph theory, computer science, robotics [21-25].

The conditions for a mapping to be nonexpansive must hold for all points in the domain of the mappings. Therefore, the need for relaxed conditions arose which do not affect the outcome of the fixed point results. In order to address this problem, Suzuki [26] introduced a new class of mappings, formally known as the class of mapping satisfying the Condition (C) in the context of uniform convex Banach spaces. The class of nonexpansive mappings is the proper subclass of the class of mapping satisfying the Condition 
(C). This class of mappings need not be continuous. Akbar and Eslamian [27] extended this class of single valued mappings to the class of multivalued mappings and obtained fixed points of such mappings in the framework of Banach spaces. These results were then obtained in the setup of complete CAT(0) spaces [28,29].

The class of $\alpha$-nonexpansive mappings in Banach spaces were suggested by Aoyama and Kohshaka [30] who also explored fixed points of such mappings. Recently, Iqbal et al. [31] proposed the concept of multivalued generalized $\alpha$-nonexpansive mappings and obtained existence and approximation results in the setting of a Banach space. In 2018, Harandi et al. [32] presented the class of $(\alpha, \beta)$-nonexpansive mappings which is properly larger than the class of $\alpha$-nonexpansive mapping for a fixed point sequence. Many researchers have presented and studied iterative techniques for approximating the fixed points and established convergence results in CAT(0) spaces for the general class of multivalued mappings including Mann, Ishikawa and $S$-iterative schemes [20,25,33,34].

Motivated by [26,32], we present the class of monotone multivalued generalized $(\alpha, \beta)$-nonexpansive multivalued mappings and establish the existence of fixed points for such mappings in the setting of an ordered CAT(0) space. We will approximate the fixed points of the proposed mapping using the $S$-iterative scheme. Under suitable conditions $\Delta$-convergence and strong convergence results will be established. An application of the convergence results is also presented. Now, we recall some important definitions and results needed in the sequel. We assume that $(Y, d)$ is a CAT $(0)$ space.

Lemma 1. [35] For $v, y \in Y$ and $q \in[0,1]$, there exists a unique $h \in[v, y]$ such that

$$
d(v, h)=(1-q) d(v, y) \text { and } d(y, h)=q d(v, y) .
$$

We denote the unique point $h \in[v, y]$ in the above Lemma by $(1-q) v \oplus q y$.

Lemma 2. [35] For $v, y, z \in Y$ and $q \in[0,1]$, we have the following inequalities:

(i) $\quad d((1-q) v \oplus q y, z) \leq(1-q) d(v, z)+q d(y, z)$.

(ii) $d((1-q) v \oplus q y, z)^{2} \leq(1-q) d(v, z)^{2}+q d(y, z)^{2}-q(1-q) d(v, y)^{2}$.

Let $M$ be a bounded subset $Y$ and $\left\{v_{n}\right\}$ a bounded sequence in $Y$ then:

(i) Define a mapping $r\left(.,\left\{v_{n}\right\}\right): Y \rightarrow \mathbb{R}^{+}$by

$$
r\left(v,\left\{v_{n}\right\}\right)=\limsup _{n \rightarrow \infty} d\left(v_{n}, v\right) .
$$

For each $v \in Y$, the value $r\left(v,\left\{v_{n}\right\}\right)$ is called asymptotic radius of $\left\{v_{n}\right\}$ at $v$ [1].

(ii) The asymptotic radius of $\left\{v_{n}\right\}[1]$ relative to $M$ is the number $r$ given by

$$
r=\inf \left\{r\left(v,\left\{v_{n}\right\}\right) ; v \in M\right\} .
$$

Denote asymptotic radius of $\left\{v_{n}\right\}$ relative to $M$ by $r\left(M,\left\{v_{n}\right\}\right)$.

(iii) The asymptotic center of $\left\{v_{n}\right\}$ relative to $M$ [1] is the set $A\left(\left\{v_{n}\right\}\right)$ of points in $Y$ for which $r\left(M,\left\{v_{n}\right\}\right)=r\left(v,\left\{v_{n}\right\}\right)$, that is,

$$
A\left(\left\{v_{n}\right\}\right)=\left\{v \in Y: r\left(v,\left\{v_{n}\right\}\right)=r\right\} .
$$

Definition 1. [35] A sequence $\left\{v_{n}\right\}$ in a $C A T(0)$ space $Y$ is $\triangle$-convergent to $v \in Y$ if $v$ is the unique asymptotic center of every subsequence of $\left\{v_{n}\right\}$. In such situation, we write $\triangle-\lim _{n} v_{n}=v$ and $v$ is the $\triangle$-limit of $\left\{v_{n}\right\}$.

Given $\left\{v_{n}\right\} \subset Y$ such that $\left\{v_{n}\right\} \triangle$-converges to $v$ if we take $y \in Y$ such that $v \neq y$, then by the uniqueness of the asymptotic center, we have $\limsup _{n \rightarrow \infty} d\left(v_{n}, v\right)<\limsup _{n \rightarrow \infty} d\left(v_{n}, y\right)$. 
Lemma 3. [35] In a complete CAT(0) space, every bounded sequence admits a $\triangle$-convergent subsequence.

Lemma 4. [35] If $\left\{v_{n}\right\}$ is a bounded sequence in a closed convex subset $M$ of a complete CAT(0) space, then the asymptotic center of $\left\{v_{n}\right\}$ is in $M$.

Lemma 5. [36] Let $p$ be an element of a complete CAT(0) space $Y$. Assuming $\left\{t_{n}\right\}$ is a sequence in $[\theta, \eta]$ for some $\theta, \eta \in(0,1)$ and that $\left\{v_{n}\right\},\left\{y_{n}\right\}$ are two sequences in $Y$ satisfying the following for some $r \geq 0$ :

$$
\begin{aligned}
\limsup _{n \rightarrow \infty} d\left(v_{n}, p\right) & \leq r, \limsup _{n \rightarrow \infty} d\left(y_{n}, p\right) \leq r \text { and } \\
\limsup _{n \rightarrow \infty} d\left(t_{n} v_{n}+\left(1-t_{n}\right) y_{n}, p\right) & =r .
\end{aligned}
$$

Then

$$
\lim _{n \rightarrow \infty} d\left(v_{n}, y_{n}\right)=0
$$

Let $M$ be a nonempty convex subset of $Y$ and $\mathcal{U}: M \longrightarrow \Omega(M)$ with $p \in F(\mathcal{U})$. Then,

(1) the Mann iterative process is defined by $x_{1} \in M$,

$$
x_{n+1}=\left(1-\alpha_{n}\right) x_{n} \oplus \alpha_{n} y_{n},
$$

where $y_{n} \in \mathcal{U} v_{n}$ satisfies

$$
d\left(y_{n+1}, y_{n}\right) \leq H\left(\mathcal{U} x_{n+1}, \mathcal{U} x_{n}\right)
$$

and $\alpha_{n} \in[0,1]$,

(2) the Ishikawa iterative process is defined as $x_{1} \in M$,

$$
y_{n}=\left(1-\beta_{n}\right) x_{n} \oplus \beta_{n} s_{n}, \quad x_{n+1}=\left(1-\alpha_{n}\right) x_{n} \oplus \alpha_{n} s_{n}^{\prime},
$$

with $s_{n} \in \mathcal{U} x_{n}$ and $s_{n}^{\prime} \in \mathcal{U} y_{n}$ satisfying $d\left(s_{n}, s_{n}^{\prime}\right) \leq H\left(\mathcal{U} x_{n}, \mathcal{U} y_{n}\right)$ and $d\left(s_{n+1}, s_{n}^{\prime}\right) \leq H\left(\mathcal{U} x_{n+1}, \mathcal{U} y_{n}\right)$, and $\alpha_{n}, \beta_{n} \in[0,1]$.

The a modification of $S$-iterative scheme [37] in the frame work of CAT(0) spaces is given as follows:

Let $v_{1} \in M$. Define

$$
\begin{aligned}
& y_{n}=\left(1-\beta_{n}\right) x_{n} \oplus \beta_{n} s_{n}, \\
& x_{n+1}=\left(1-\alpha_{n}\right) s_{n} \oplus \alpha_{n} z_{n}^{\prime},
\end{aligned}
$$

with $s_{n} \in \mathcal{U} x_{n}, s_{n}^{\prime} \in \mathcal{U} y_{n}$ and $d\left(s_{n}, s_{n}^{\prime}\right) \leq H\left(\mathcal{U} x_{n}, \mathcal{U} y_{n}\right)$ satisfying $d\left(s_{n+1}, s_{n}^{\prime}\right) \leq H\left(\mathcal{U} x_{n+1}, \mathcal{U} y_{n}\right)$ and $\alpha_{n}, \beta_{n} \in(0,1)$.

Consider a complete CAT(0) space, $Y$, endowed with partial order $\preceq$. Two elements $v, y$ are comparable if $v \preceq y$ or $y \preceq v$. For any $a \in Y$, define

$$
[a, \rightarrow)=\{v \in Y ; a \preceq v\} \text { and }(\leftarrow, a]=\{v \in Y ; v \preceq a\}
$$

Let $v, y \in Y$. An order interval $[v, y]$ is the set given by

$$
[v, y]=\{w \in Y: v \preceq w \preceq y\}
$$

Let $M$ be a nonempty closed convex subset of $(Y, \preceq)$. A mapping $\mathcal{U}: M \longrightarrow \Omega(M)$ is called monotone if for any $u_{v} \in \mathcal{U} v$ there exists $u_{y} \in \mathcal{U} y$ such that $u_{v} \preceq u_{y}$ whenever $v \preceq y$ for all $v, y \in M$. Moreover, the mapping $\mathcal{U}$ is:

(i) monotone nonexpansive if $\mathcal{U}$ is monotone and such that for any comparable $v, y \in M$,

$$
H(\mathcal{U}, \mathcal{U} y) \leq d(v, y)
$$


(ii) monotone quasi-nonexpansive if $\mathcal{U}$ is monotone with $p \in F(\mathcal{U}) \neq \varnothing$ and $v \in M$ such that whenever $v, p$ are comparable,

$$
H(\mathcal{U} v, \mathcal{U} p) \leq d(v, p)
$$

holds.

Throughout this paper, we consider the order intervals to be closed convex subsets of an ordered $\operatorname{CAT}(0)$ space $(Y, \preceq)$.

\section{Multivalued Generalized $(\alpha, \beta)$-Nonexpansive Mapping}

In this section, we introduce a class of multivalued generalized $(\alpha, \beta)$-nonexpansive mapping in the setting of CAT(0) spaces which is a wider class of nonexpansive type mapping which properly contains nonexpansive, mappings satisfying the Condition (C) and generalized $\alpha$-nonexpansive mappings. We also discuss some of its properties in CAT(0) space.

Definition 2. Let $M$ be a nonempty subset of a $C A T(0)$ space $(Y, d)$. A multivalued mapping $\mathcal{U}: M \longrightarrow \Omega(Y)$ satisfies the Condition $\left(C_{(\alpha, \beta)}\right)$ if there exists $\alpha, \beta \in(0,1)$ such that for any $v, y \in M$,

$$
\begin{aligned}
\frac{1}{2} d(v, \mathcal{U}) & \leq d(v, y) \quad \text { implies that } \\
H(\mathcal{U}, \mathcal{U} y) & \leqslant \alpha d(y, \mathcal{U} v)+\beta d(v, \mathcal{U} y)+(1-\alpha-\beta) d(v, y)
\end{aligned}
$$

If a multivalued mapping satisfies the Condition $\left(C_{(\alpha, \beta)}\right)$ in a $\operatorname{CAT}(0)$ space then we say $\mathcal{U}$ is the multivalued generalized $(\alpha, \beta)$-nonexpansive mapping.

Let $M$ be a nonempty closed subset of an ordered CAT $(0)$ space $(Y, \preceq)$. A mapping $\mathcal{U}: M \longrightarrow \Omega(M)$ is said to be a monotone multivalued generalized $(\alpha, \beta)$ - nonexpansive mapping if
(a) $U$ is monotone,
(b) $\mathcal{U}$ satisfies (9) for all $v, y \in M$ and either $v \preceq y$ or $y \preceq v$.

\section{Remark 1.}

(1) Multivalued generalized $(\alpha, \beta)$-nonexpansive mappings extend and generalize the class of mappings introduced by [31]. Indeed, if $\alpha=\beta$ then the mapping is reduced to multivalued generalized $\alpha$-nonexpansive mapping.

(2) Multivalued generalized $(\alpha, \beta)$-nonexpansive mappings contain the class of mappings satisfying the Condition (C). Clearly, substituting $\alpha=\beta=0$ we get our desired mapping.

(3) Every nonexpansive mapping is generalized $(0,0)$-nonexpansive mapping.

We present an example of a multivalued generalized $(\alpha, \beta)$-nonexpansive mappings in an ordered CAT(0) space which is neither nonexpansive or satisfies the Condition (C).

Example 1. Consider an Example 18 of [38] where

$$
d(v, y)=\left|v_{1}-y_{1}\right|+\left|v_{1} v_{2}-y_{1} y_{2}\right|
$$

Define an order on $Y$ as follows: for $v=\left(v_{1}, v_{2}\right)$ and $y=\left(y_{1}, y_{2}\right), v<y$ if and only if $v_{1} \leq y_{1}$ and $v_{2} \leq y_{2}$. Thus $(Y, d, \preceq)$ is an ordered Hyperbolic space which is an example of an ordered $C A T(0)$ space.

Let $M=[0,2] \times[0,2] \subset Y$ and $\mathcal{U}: M \longrightarrow \Omega(M)$ be defined by

$$
\mathcal{U}\left(v_{1}, v_{2}\right)= \begin{cases}\left\{\left(0, \frac{1}{4}\right),\left(\frac{1}{2}, 1\right)\right\}, & \text { if }\left(v_{1}, v_{2}\right) \neq(2,2), \\ \left\{\left(\frac{3}{2}, \frac{3}{2}\right),\left(\frac{19}{10}, \frac{19}{10}\right)\right\}, & \text { if } \quad\left(v_{1}, v_{2}\right)=(2,2) .\end{cases}
$$


(1) The mapping $\mathcal{U}$ does not satisfy the Condition (C) on $M$ and therefore is not nonexpansive. Indeed, for $\left(v_{1}, v_{2}\right)=(1,1)$ and $\left(y_{1}, y_{2}\right)=(2,2)$ we have

$$
\frac{1}{2} d(v, \mathcal{U} v)=\frac{1}{2} \min \left\{\frac{1}{2}, \frac{1}{4}\right\}=\frac{1}{8}
$$

and

$$
d(v, y)=|1-2|+|1(1)-2(2)|=4 \text {. }
$$

Thus, $\frac{1}{2} d(v, \mathcal{U} v)<d(v, y)$. Note that

$$
\operatorname{dist}(z, \mathcal{U} y)=\inf \left\{\left|s_{1}-\frac{3}{2}\right|+\left|s_{1} z_{2}-\frac{3}{2} \cdot \frac{3}{2}\right|,\left|s_{1}-\frac{3}{2}\right|+\left|s_{1} z_{2}-\frac{19}{10} \cdot \frac{19}{10}\right|\right\}
$$

If $z \in \mathcal{U}$ v then we have

$$
\begin{aligned}
\sup _{z \in \mathcal{U}_{v}} \operatorname{dist}(z, \mathcal{U}(y) & =\sup \left\{\inf \left\{\frac{15}{4}, \frac{551}{100}\right\}, \inf \left\{\frac{11}{4}, \frac{451}{100}\right\}\right\} \\
& =\sup \left\{\frac{15}{4}, \frac{11}{4}\right\}=\frac{15}{4}
\end{aligned}
$$

Also,

$$
\operatorname{dist}(w, \mathcal{U} v)=\inf \left\{\left|w_{1}\right|+\left|w_{1} w_{2}\right|,\left|w_{1}-\frac{1}{2}\right|+\left|w_{1} w_{2}-\frac{1}{2}\right|\right\}
$$

If $w \in \mathcal{U} y$, then we have

$$
\begin{aligned}
\sup _{w \in \mathcal{U} y} \operatorname{dist}(w, \mathcal{U} v) & =\sup \left\{\inf \left\{\frac{15}{4}, \frac{11}{4}\right\}, \inf \left\{\frac{551}{100}, \frac{451}{100}\right\}\right\} \\
& =\sup \left\{\frac{11}{4}, 4.51\right\}=4.51 .
\end{aligned}
$$

Thus

$$
H(\mathcal{U}, \mathcal{U} y)=\max \left\{\frac{15}{4}, \frac{451}{100}\right\}=4.51
$$

implies that $H(\mathcal{U} v, \mathcal{U} y)>d(v, y)$.

(2) Now, we show that $\mathcal{U}$ is multivalued generalized $(\alpha, \beta)$-nonexpansive mapping, where $\alpha=\frac{7}{8}$ and $\beta=\frac{1}{8}$. We consider the following cases.

CASE - I If $v=\left(v_{1}, v_{2}\right) \neq(2,2)$ and $y=\left(y_{1}, y_{2}\right)=(2,2)$, Observe that

$$
\begin{aligned}
\operatorname{dist}(y, u v) & =\inf \left\{d\left(\left(y_{1}, y_{2}\right),\left\{\left(0, \frac{1}{4}\right),\left(\frac{1}{2}, 1\right)\right\}\right)\right\} \\
& ==\frac{11}{2}
\end{aligned}
$$

and

$$
\begin{aligned}
\operatorname{dist}(v, \mathfrak{u} y) & =\inf \left\{d\left(\left(v_{1}, v_{2}\right),\left\{\left(\frac{3}{2}, \frac{3}{2}\right),\left(\frac{19}{10}, \frac{19}{10}\right)\right\}\right\}\right. \\
& =\left|v_{1}-\frac{3}{2}\right|+\left|v_{1} v_{2}-\frac{9}{4}\right| .
\end{aligned}
$$


For $\alpha=\frac{7}{8}$ and $\beta=\frac{1}{8}$, we obtain that

$$
\begin{aligned}
\alpha \operatorname{dist}(y, \mathcal{U}) & +\beta \operatorname{dist}(v, \mathcal{U} y)+(1-\alpha-\beta) d(v, y) \\
& =\frac{7}{8} \cdot \frac{11}{2}+\frac{1}{8}\left(\left|v_{1}-\frac{3}{2}\right|+\left|v_{1} v_{2}-\frac{9}{4}\right|\right)+\left(1-\frac{7}{8}-\frac{1}{8}\right) d(v, y) \\
& \geq \frac{1}{8}\left|v_{1}-\frac{3}{2}-v_{1} v_{2}+\frac{9}{4}\right|+\frac{77}{16} \geq 4.51=H\left(\mathcal{U}, U_{y}\right) .
\end{aligned}
$$

CASE - II If $v=\left(v_{1}, v_{2}\right)=y=\left(y_{1}, y_{2}\right) \neq(2,2)$, then

$$
\begin{aligned}
\operatorname{dist}(z, \mathcal{U} y) & =\inf \left\{d\left(\left(s_{1}, z_{2}\right),\left\{\left(0, \frac{1}{4}\right),\left(\frac{1}{2}, 1\right)\right\}\right)\right\} \\
& =\inf \left\{\left|s_{1}\right|+\left|s_{1} z_{2}\right|,\left|s_{1}-\frac{1}{2}\right|+\left|s_{1} z_{2}-\frac{1}{2}\right|\right\}
\end{aligned}
$$

Now $z \in \mathcal{U}$ gives that

$$
\sup _{z \in \mathcal{U}_{v}} \operatorname{dist}(z, \mathcal{U} y)=\sup \{\inf \{0,1\}, \inf \{1,0\}\}=\sup \{0,0\}=0 .
$$

Similarly,

$$
\operatorname{dist}(w, \mathcal{U} v)=\inf \left\{\left|w_{1}\right|+\left|w_{1} w_{2}\right|,\left|w_{1}-\frac{1}{2}\right|+\left|w_{1} w_{2}-\frac{1}{2}\right|\right\}
$$

Now w $\in$ Uy gives

$$
\sup _{w \in \mathcal{U} y} \operatorname{dist}(w, \mathcal{U} v)=\sup \{\inf \{0,1\}, \inf \{1,0\}\}=0
$$

Thus

$$
H(\mathcal{u}, \mathcal{U} y)=0
$$

Also,

$$
\begin{aligned}
\operatorname{dist}(v, \mathcal{U} y) & =\left|v_{1}-\frac{1}{2}\right|+\left|v_{1} v_{2}-\frac{1}{2}\right| \\
\text { and } & \\
\operatorname{dist}(y, \mathcal{U} v) & =\left|y_{1}-\frac{1}{2}\right|+\left|y_{1} y_{2}-\frac{1}{2}\right| .
\end{aligned}
$$

For $\alpha=\frac{7}{8}$ and $\beta=\frac{1}{8}$, we obtain that

$$
\begin{aligned}
\alpha \operatorname{dist}(y, \mathcal{U} v) & +\beta \operatorname{dist}(v, \mathcal{U} y)+(1-\alpha-\beta) d(v, y) \mid \\
& =\frac{7}{8}\left(\left|y_{1}-\frac{1}{2}\right|+\left|y_{1} y_{2}-\frac{1}{2}\right|\right)+\frac{1}{8}\left(\left|v_{1}-\frac{1}{2}\right|+\left|v_{1} v_{2}-\frac{1}{2}\right|\right)+\left(1-\frac{7}{8}-\frac{1}{8}\right) d(v, y) \\
& \geq \frac{1}{8}\left|v_{1}-v_{1} v_{2}-7 y_{1}-7 y_{1} y_{2}-\frac{3}{4}\right| \\
& \geq 0=H\left(U_{v}, U_{y}\right) .
\end{aligned}
$$

Hence $\mathcal{U}$ is $\left(\frac{7}{8}, \frac{1}{8}\right)$-nonexpansive multivalued mapping.

Proposition 1. Let $\mathcal{U}: M \longrightarrow \Omega(M)$ be a multivalued mapping then the following hold.

(1) If $\mathcal{U}$ satisfies the Condition (C) as defined in (3), then $\mathcal{U}$ satisfies the Condition $\left(C_{(\alpha, \beta)}\right)$.

(2) If $U$ satisfies the Condition $\left(C_{(\alpha, \beta)}\right)$ with $F(\mathcal{U}) \neq \varnothing$, then $\mathcal{U}$ is quasi-nonexpansive. 


\section{Proof.}

(1) If $\mathcal{U}$ satisfies the Condition (C) then it trivial that $\mathcal{U}$ satisfies the Condition $\left(C_{(\alpha, \beta)}\right)$ for $\alpha=\beta=0$.

(2) Let $p \in F(\mathcal{U}) \neq \varnothing$ then,

$$
\frac{1}{2} \operatorname{dist}(p, \mathcal{U} p)=0 \leq d(v, p) \text { for all } v \in Y
$$

As $\mathcal{U}$ satisfies the Condition $\left(C_{(\alpha, \beta)}\right)$, there exists $\alpha, \beta \in[0,1)$ such that

$$
H(\mathcal{U} v, \mathcal{U} p) \leq \alpha \operatorname{dist}(p, \mathcal{U} v)+\beta \operatorname{dist}(v, \mathcal{U} p)+(1-\alpha-\beta) d(v, p)
$$

holds. Then,

$$
H(\mathcal{U} v, \mathcal{U} y) \leq \alpha H(\mathcal{U} v, \mathcal{U} p)+\beta \operatorname{dist}(p, \mathcal{U} p)+\beta d(v, p)+(1-\alpha-\beta) d(v, p)
$$

implies

$$
(1-\alpha) H(\mathcal{U} v, \mathcal{U} p) \leq(1-\alpha) d(v, p) .
$$

Since $1-\alpha>0$, it follows that $H(\mathcal{U} v, \mathcal{U} p) \leq d(v, p)$. Hence $\mathcal{U}$ is quasi-nonexpansive multivalued mapping.

Remark 2. The converse of (i) in the Proposition 1 is not true in general. Indeed if a multivalued mapping satisfies the Condition $\left(C_{(\alpha, \beta)}\right)$, it does not necessarily imply that the mapping satisfies the Condition (C).

Now, we characterize some properties of $F(\mathcal{U})$ of multivalued mapping in CAT(0) space. For the following result sin this section, assume that $M$ is a nonempty subset of a CAT(0) space $Y$ and $\mathcal{U}: M \longrightarrow \Omega(M)$ a multivalued mapping satisfying the Condition $\left(C_{(\alpha, \beta)}\right)$ for some $\alpha, \beta \in[0,1)$,

Theorem 1. If $M$ is closed then $F(\mathcal{U})$ is closed. Moreover, if $M$ is convex and $F(\mathcal{U}) \neq \varnothing$ with $S F(\mathcal{U})=F(\mathcal{U})$, then $F(\mathcal{U})$ is convex.

Proof. Let $\left\{p_{n}\right\}$ be a sequence in $F(\mathcal{U})$ such that $\lim _{n \rightarrow \infty} d\left(p_{n}, p\right)=0$ for some $p \in M$. Since

$$
\frac{1}{2} \operatorname{dist}\left(p_{n}, \mathcal{U} p_{n}\right)=0 \leq d\left(p_{n}, p\right) \text { for all } p \in M
$$

and $\mathcal{U}$ satisfies the Condition $\left.\left(C_{(\alpha, \beta}\right)\right)$, there exists $\alpha, \beta \in[0,1)$ such that

$$
H\left(\mathcal{U} p_{n}, \mathcal{U} p\right) \leq \alpha \operatorname{dist}\left(p, \mathcal{U} p_{n}\right)+\beta \operatorname{dist}\left(p_{n}, \mathcal{U} p\right)+(1-\alpha-\beta) d\left(p_{n}, p\right) \quad \forall p \in M .
$$

Thus

$$
\operatorname{dist}\left(p_{n}, \mathcal{U} p\right) \leq H\left(\mathcal{U} p_{n}, \mathcal{U} p\right)
$$

and

$$
H\left(\mathcal{U} p_{n}, \mathcal{U} p\right) \leq \alpha \operatorname{dist}\left(p, \mathcal{U} p_{n}\right)+\beta \operatorname{dist}\left(p_{n}, \mathcal{U} p\right)+(1-\alpha-\beta) d\left(p_{n}, p\right)
$$

imply that

$$
(1-\beta) \operatorname{dist}\left(p_{n}, \mathcal{U} p\right) \leq(1-\beta) d\left(p_{n}, p\right) .
$$

As $1-\beta>0$, on taking the limit on both sides, we obtain that $d(p, \mathcal{U} p)=0$. Therefore $p \in \mathcal{U} p$ and hence $F(\mathcal{U})$ is closed. 
Let $v, y \in F(\mathcal{U})$. We need to show that every geodesic segment joining $v$ and $y$ lies in $F(\mathcal{U})$. Let $h \in[v, y]$ with $h=t v \oplus(1-t) y$ for some $t \in[0,1]$. Since $M$ is convex and $h \in[v, y] \subset M$. Let $p \in \mathcal{U} p$. Then by Lemma 2 and $\frac{1}{2} d(p, \mathcal{U} p)=0$, we have

$$
\begin{aligned}
d(h, p)^{2} & =d(t v \oplus(1-t) y, p)^{2} \\
& \leq t d(v, p)^{2}+(1-t) d(y, p)^{2}-t(1-t) d(v, y)^{2} \\
& =t \operatorname{dist}(p, \mathcal{U})^{2}+(1-t) \operatorname{dist}(p, \mathcal{U} y)^{2}-t(1-t) d(v, y)^{2} \\
& \leq t H(\mathcal{U}, \mathcal{U} v)^{2}+(1-t) H(\mathcal{U}, \mathcal{U} y)^{2}-t(1-t) d(v, y)^{2} \\
& \leq t(\alpha \operatorname{dist}(v, \mathcal{U} p)+\beta \operatorname{dist}(p, \mathcal{U} v)+(1-\alpha-\beta) d(v, p))^{2} \\
& +(1-t)(\alpha \operatorname{dist}(y, \mathcal{U} p)+\beta \operatorname{dist}(p, \mathcal{U} y)+(1-\alpha-\beta) d(y, p))^{2}-t(1-t) d(v, y)^{2} .
\end{aligned}
$$

Further, we have

$$
\begin{aligned}
d(h, p)^{2} & \leq t(\alpha d(v, p)+\beta d(v, p)+(1-\alpha-\beta) d(v, p))^{2}+ \\
& (1-t)(\alpha d(y, p)+\beta d(y, p)+(1-\alpha-\beta) d(y, p))^{2}-t(1-t) d(v, y)^{2} \\
& \leq t d(v, p)^{2}+(1-t) d(y, p)^{2}-t(1-t) d(v, y)^{2} .
\end{aligned}
$$

Now by using Lemma 2, we have

$$
\begin{aligned}
d(h, p)^{2} & \leq t(1-t)^{2} d(v, y)^{2}+t^{2}(1-t) d(v, y)^{2}-t(1-t) d(v, y)^{2} \\
& =0 .
\end{aligned}
$$

This implies that $h=p \in \mathcal{U} p$, that is, $p \in F(\mathcal{U})$. Hence $F(\mathcal{U})$ is convex.

Now, we present some results associated with the multivalued mapping satisfying the Condition $\left(C_{(\alpha, \beta)}\right)$.

Proposition 2. For each $v, y \in M$ and $p \in \mathcal{U} v$ we have the following:

(1) $H(\mathcal{U} v, \mathcal{U} p) \leq d(v, p)$.

(2) Either $\frac{1}{2} \operatorname{dist}(v, \mathcal{U} v) \leq d(v, y)$ or $\frac{1}{2} \operatorname{dist}(p, \mathcal{U} p) \leq d(y, p)$.

(3) Either $H\left(\mathcal{U}, \mathcal{U}_{y}\right) \leq \alpha \operatorname{dist}(y, \mathcal{U} v)+\beta \operatorname{dist}(v, \mathcal{U} y)+(1-\alpha-\beta) d(v, y)$ or $H(\mathcal{U} v, \mathcal{U} p) \leq \alpha \operatorname{dist}(p, \mathcal{U} v)+\beta \operatorname{dist}(v, \mathcal{U} p)+(1-\alpha-\beta) d(v, p)$.

\section{Proof.}

(1) As, $\frac{1}{2} \operatorname{dist}\left(v, \mathcal{U v}_{v}\right) \leq \operatorname{dist}\left(v, \mathcal{U}_{v}\right) \leq d(v, p) \forall p \in \mathcal{U}_{v}$, we have

$$
H(\mathcal{U}, \mathcal{U} p) \leq \alpha \operatorname{dist}(p, \mathcal{U} v)+\beta \operatorname{dist}(v, \mathcal{U} p)+(1-\alpha-\beta) d(v, p)
$$

which implies that

$$
H\left(U_{v}, U_{p}\right) \leq \alpha d(v, p)+\alpha H\left(U_{v}, U_{p}\right)+\beta d(v, p)+(1-\alpha-\beta) d(v, p)
$$

and hence

$$
H(\mathcal{U}, \mathcal{U} p) \leq d(v, p)
$$

we have

$$
H(\mathcal{U}, \mathcal{U} p) \leq \alpha \operatorname{dist}(p, \mathcal{U} v)+\beta \operatorname{dist}(v, \mathcal{U} p)+(1-\alpha-\beta) d(v, p)
$$

which implies that

$$
H(\mathcal{U} v, \mathfrak{U} p) \leq \alpha d(v, p)+\alpha H\left(U_{v}, \mathfrak{U} p\right)+\beta d(v, p)+(1-\alpha-\beta) d(v, p)
$$


and hence

$$
H(\mathcal{U} v, \mathcal{U} p) \leq d(v, p) .
$$

(2) Suppose that $\frac{1}{2} \operatorname{dist}(v, \mathcal{U} v)>d(v, y)$ and $\frac{1}{2} \operatorname{dist}(p, \mathcal{U} p)>d(y, p)$. From (i), we get

$$
\begin{aligned}
\frac{1}{2} \operatorname{dist}(p, \mathcal{U} p) & \leq \operatorname{dist}(p, \mathcal{U} p) \leq d(v, p) \text { and } \\
\operatorname{dist}(p, \mathcal{U} p) & \leq d(v, y)+(y, p) \\
& <\frac{1}{2} \operatorname{dist}(v, \mathcal{U} v)+\frac{1}{2} \operatorname{dist}(p, \mathcal{U} p) .
\end{aligned}
$$

Thus,

$$
\operatorname{dist}(p, \mathcal{U} p)-\frac{1}{2} \operatorname{dist}(p, \mathcal{U} p)<\frac{1}{2} \operatorname{dist}(v, \mathcal{U} v)
$$

implies that

$$
\operatorname{dist}(p, \mathcal{U} p)<\operatorname{dist}(v, \mathcal{U} v)
$$

Also, we have

$$
\begin{aligned}
\operatorname{dist}(v, \mathcal{U} v) & \leq d(v, p) \\
& \leq d(v, y)+d(y, p) \\
& <\frac{1}{2} \operatorname{dist}(v, \mathcal{U} v)+\frac{1}{2} \operatorname{dist}(p, \mathcal{U} p) .
\end{aligned}
$$

Thus,

$$
\begin{aligned}
\operatorname{dist}(v, \mathcal{U} v)-\frac{1}{2} \operatorname{dist}(v, \mathcal{U} v) & <\frac{1}{2} \operatorname{dist}(p, \mathcal{U} p) \\
\text { implies that } \operatorname{dist}(v, \mathcal{U} v) & <\operatorname{dist}(p, \mathcal{U} p)
\end{aligned}
$$

Combining the inequalities (12) and (13), we obtain $\operatorname{dist}(v, \mathcal{U} v)<\operatorname{dist}(v, \mathcal{U v})$ a contradiction. Hence (ii) holds.

(3) The condition (iii) directly follow from the condition (ii).

Proposition 3. Let $M$ be closed and convex then

$$
H(\mathcal{U} v, \mathcal{U} y) \leq \frac{(2+\alpha+\beta)}{(1-\beta)} \operatorname{dist}(v, \mathcal{U} v)+d(v, y)
$$

holds for all $v, y \in M$.

Proof. Let $v \in M$, then there exists $p \in \mathcal{U} v$ such that $d(v, p)=\operatorname{dist}(v, \mathcal{U} v)$. By using Proposition 2, we have

$$
H(\mathcal{U} v, \mathcal{U} p) \leq d(v, p) .
$$

Now, by proposition 3.2 (iii), we obtain

$$
H(\mathcal{U} v, \mathcal{U} y) \leq \alpha \operatorname{dist}(y, \mathcal{U} v)+\beta \operatorname{dist}(v, \mathcal{U} y)+(1-\alpha-\beta) d(v, y),
$$

or

$$
H(\mathcal{U} p, \mathcal{U} y) \leq \alpha \operatorname{dist}(y, \mathcal{U} p)+\beta \operatorname{dist}(p, \mathcal{U} y)+(1-\alpha-\beta) d(y, p)
$$


If inequality (16) holds, then we get

$$
\begin{aligned}
H(U v, U y) & \leq \alpha \operatorname{dist}(y, U v)+\beta \operatorname{dist}(v, \mathcal{U} y)+(1-\alpha-\beta) d(v, y) \\
& \leq \alpha d(v, y)+\alpha \operatorname{dist}(v, \mathcal{U} v)+\beta \operatorname{dist}(v, \mathcal{U} v)+\beta \operatorname{dist}\left(\mathcal{U v}_{v}, U_{y}\right)+(1-\alpha-\beta) d(v, y) \\
& \leq(\alpha+\beta) \operatorname{dist}(v, \mathcal{U})+(1-\beta) d(v, y)+\beta H(\mathcal{U}, \mathcal{U} y) \\
& \leq \frac{(\alpha+\beta)}{(1-\beta)} \operatorname{dist}(v, \mathcal{U} v)+d(v, y) .
\end{aligned}
$$

If (17) holds, then we have

$$
\begin{aligned}
& H\left(\mathcal{U}, U_{y}\right) \leq H(\mathcal{U}, \mathcal{U} p)+H\left(\mathcal{U}, \mathcal{U y}_{y}\right) \\
& \leq d(v, p)+\alpha \operatorname{dist}(y, U p)+\beta \operatorname{dist}(p, \mathcal{U} y)+(1-\alpha-\beta) d(y, p) \\
& \leq d(v, p)+\alpha d(v, y)+\alpha \operatorname{dist}(v, \mathfrak{U} p)+\beta d(v, p)+\beta \operatorname{dist}(v, \mathfrak{U} y)+ \\
& (1-\alpha-\beta) d(y, p) \\
& \leq(1+\beta) d(v, p)+\alpha d(v, y)+\alpha \operatorname{dist}(v, \mathcal{U} v)+\alpha \operatorname{dist}(\mathcal{U} v, \mathcal{U} p)+\beta \operatorname{dist}(v, \mathcal{U} v) \\
& +\beta d i s t(\mathcal{U}, \mathfrak{U} y)+(1-\alpha-\beta) d(y, p) \\
& \leq(1+\beta) d(v, p)+\alpha d(v, y)+(\alpha+\beta) \operatorname{dist}(v, \mathcal{U v})+\alpha H(\mathcal{U}, \mathcal{U} p)+ \\
& \beta H(\mathcal{U v}, \mathcal{U} y)+(1-\alpha-\beta) d(y, p)
\end{aligned}
$$

Thus

$$
\begin{aligned}
(1-\beta) H(\mathcal{U}, \mathcal{U} y) & \leq(1+\alpha+\beta) d(v, p)+\alpha d(v, y)+(\alpha+\beta) \operatorname{dist}(v, \mathcal{U v})+(1-\alpha-\beta) d(y, p) \\
& \leq(1+\alpha+\beta) d(v, p)+\alpha d(v, y)+(\alpha+\beta) \operatorname{dist}(v, \mathcal{U v})+ \\
& (1-\alpha-\beta)(d(v, p)+d(v, y))
\end{aligned}
$$

implies that

$$
H\left(U v, U_{y}\right) \leq \frac{(2+\alpha+\beta)}{(1-\beta)} \operatorname{dist}(v, \mathcal{U})+d(v, y) .
$$

Hence our desired inequality is proved in both cases.

\section{Convergence Results}

In this section, we present some existence result of fixed point of a multivalued generalized $(\alpha, \beta)$ - nonexpansive multivalued mappings in the frame work of ordered $\operatorname{CAT}(0)$ space $(Y, \preceq)$.

Lemma 6. Let $M$ be a nonempty, closed and convex subset of a complete ordered CAT(0) space $(Y, \preceq)$ and $U: M \longrightarrow \Omega(M)$ be a monotone multivalued generalized $(\alpha, \beta)-$ nonexpansive mapping. Then $\frac{1}{2} d(p, \mathcal{U} v) \leq d(p, v)$ for all $v \in M$ and $p \in F(\mathcal{U})$ such that either $v \preceq p$ or $p \preceq v$.

Lemma 7. Let $M$ and $\mathcal{U}: M \longrightarrow \Omega(M)$ be as in Lemma 6. Fix $x_{1} \in M$ such that $x_{1} \preceq s_{1}$ or $\left(s_{1} \preceq x_{1}\right)$. If $\left\{x_{n}\right\}$ is defined by (6), then we have:

(1) $\quad x_{n} \preceq s_{n} \preceq x_{n+1}$ or $x_{n+1} \preceq s_{n} \preceq x_{n}$ for any $n \geq 1$ and $s_{n} \in U\left(x_{n}\right.$.

(2) $x_{n} \preceq x$ provided that $\left\{x_{n}\right\} \triangle$-converges to a point $x \in M$.

\section{Proof.}

(1) If $x_{1} \preceq s_{1}$, then by convexity of order interval $\left[x_{1}, s_{1}\right]$ and (6) we have,

$$
x_{1} \preceq\left(1-\beta_{1}\right) x_{1} \oplus \beta_{1} s_{1} \preceq s_{1} .
$$

Thus, there exists $y_{1}$ such that

$$
x_{1} \preceq y_{1} \preceq s_{1} .
$$


As, $\mathcal{U}$ is monotone there exists $s_{1}^{\prime} \in \mathcal{U} y_{1}$ such that $s_{1} \preceq s_{1}^{\prime}$. Again by convexity of order interval $\left[s_{1}, s_{1}^{\prime}\right]$ and (6) we have

$$
s_{1} \preceq\left(1-\alpha_{1}\right) s_{1} \oplus \alpha z_{2}^{\prime} \preceq z_{2}^{\prime} .
$$

Thus,

$$
s_{1} \preceq x_{2} \preceq s_{1}^{\prime}
$$

From (18) and (19), we have

$$
x_{1} \preceq y_{1} \preceq s_{1} \preceq x_{2} \preceq z_{2}^{\prime}
$$

which implies that

$$
x_{1} \preceq s_{1} \preceq x_{2} .
$$

Hence the statement is true for $n=1$.

Assuming the statement is true for $n$, that is, for $s_{n} \in \mathcal{U} x_{n}$, we have

$$
x_{n} \preceq s_{n} \preceq x_{n+1} .
$$

Now, we show (21) is true for $n+1$.

By the convexity of order interval $\left[x_{n}, s_{n}\right]$ and (6), we have

$$
x_{n} \preceq\left(1-\beta_{n}\right) x_{n} \oplus \beta_{n} s_{n} \preceq s_{n} .
$$

Thus, we have

$$
x_{n} \preceq y_{n} \preceq s_{n} .
$$

By monotonicity of $\mathcal{U}$, there exists $s_{n}^{\prime} \in \mathcal{U} y_{n}$ such that $s_{n} \preceq s_{n}^{\prime}$. Again by convexity of order interval $\left[s_{n}, s_{n}^{\prime}\right]$ and (6) we have

$$
s_{n} \preceq\left(1-\alpha_{n}\right) s_{n} \oplus \alpha_{n} s_{n}^{\prime} \preceq s_{n}^{\prime}
$$

which implies that

$$
s_{n} \preceq x_{n+1} \preceq s_{n}^{\prime} .
$$

It follows from (22) and (23) that

$$
x_{n} \preceq y_{n} \preceq s_{n} \preceq x_{n+1} \preceq s_{n}^{\prime}
$$

and therefore

$$
s_{n}^{\prime} \preceq s_{n+1} .
$$

From (24), we have

$$
x_{n+1} \preceq s_{n+1} .
$$

By the convexity of order interval $\left[x_{n+1}, s_{n+1}\right]$ and (6), we obtain that

$$
x_{n+1} \preceq\left(1-\beta_{n+1}\right) x_{n+1} \oplus \beta_{n+1} s_{n+1} \preceq s_{n+1}
$$

and hence

$$
x_{n+1} \preceq y_{n+1} \preceq s_{n+1} .
$$

The monotonicity of $\mathcal{U}$ yields that there exists $s_{n+1}^{\prime} \in \mathcal{U} y_{n+1}$ such that $s_{n+1} \preceq s_{n+1}^{\prime}$. Now the convexity of order interval $\left[s_{n+1}, s_{n+1}^{\prime}\right]$ and (6) gives that

$$
s_{n+1} \preceq\left(1-\alpha_{n+1}\right) s_{n+1} \oplus \alpha_{n+1} s_{n+1}^{\prime} \preceq s_{n+1}^{\prime}
$$

which implies that

$$
s_{n+1} \preceq x_{n+2} \preceq s_{n+1}^{\prime}
$$


So, from (25) and (26), we obtain

$$
x_{n+1} \preceq s_{n+1} \preceq x_{n+2} .
$$

Hence, (21) is true for all $n$.

(2) Suppose that $x$ is $\triangle$-limit of $\left\{x_{n}\right\}$. From (i), we have $x_{n} \preceq x_{n+1}$ for all $n \geq 1$. Since the order interval $\left[x_{m}, \rightarrow\right)$ is closed and convex and the sequence is $\left\{x_{n}\right\}$ is increasing we deduce that $x \in\left[x_{m}, \rightarrow\right)$ for a fixed $m \in \mathbb{N}$, if not, that is, if $x \notin\left[x_{m}, \rightarrow\right)$, then a subsequence $\left\{x_{k}\right\}$ of $\left\{x_{n}\right\}$ may be constructed by leaving the first $m-1$ terms of the sequence $\left\{x_{n}\right\}$, and then the asymptotic center of $\left\{x_{r}\right\}$ would not be $x$, which contradicts that $x$ is the $\triangle$-limit of the sequence $\left\{x_{n}\right\}$. This completes the proof.

Lemma 8. Let $M$ and $\mathcal{U}: M \longrightarrow \Omega(M)$ be as in Lemma 6 and $\left\{x_{n}\right\}$ be a sequence defined by (6) where $F(\mathcal{U}) \neq \varnothing$ such that $S F(\mathcal{U})=F(\mathcal{U})$. Suppose that there exists $x_{1} \in M$ such that $x_{1} \preceq s_{1}$, where $s_{1} \in U x_{1}$. Also, assume that either $x_{1}$ and $p$ are comparable then

(1) $\lim _{n \rightarrow \infty} d\left(x_{n}, p\right)$ exists for all $p \in F(\mathcal{U})$.

(2) $\lim _{n \rightarrow \infty} d\left(x_{n}, s_{n}\right)=0$ where $s_{n} \in \mathcal{U} x_{n}$.

\section{Proof.}

(1) Let $p \in F(\mathcal{U})$. If $p \preceq x_{1}$, then Lemma 7 and the transitivity of the order imply $p \preceq x_{2}$. Applying mathematical induction, we obtain $p \preceq x_{n}$ for all $n \geq 1$. On the other hand, assume that $x_{1} \preceq p$. Since there exists $s_{1} \in U x_{1}$ we have $s_{1} \preceq p$ as $F(\mathcal{U})=S F(\mathcal{U})$. Further, (21) yields

$$
y_{1}=\left(1-\beta_{1}\right) x_{1} \oplus \beta_{1} s_{1} \preceq p .
$$

Again, there exists $s_{1}^{\prime} \in \mathcal{U} y_{1}$ which implies that $s_{1}^{\prime} \preceq p$ as $F(\mathcal{U})=S F(\mathcal{U})$. Finally, we have

$$
x_{2}=\left(1-\alpha_{1}\right) s_{1} \oplus \alpha_{1} s_{1}^{\prime} \preceq p .
$$

Continuing in this manner, we obtain $y_{n} \preceq p, s_{n}^{\prime} \preceq p$ and $x_{n} \preceq p$. Therefore, in both cases $x_{n}$ and $p$ are comparable. Now, from (21) we have

$$
\begin{aligned}
d\left(x_{n+1}, p\right) & =d\left(\left(1-\alpha_{n}\right) s_{n}+\alpha_{n} s_{n}^{\prime}, p\right) \\
& \leq\left(1-\alpha_{n}\right) d\left(s_{n}, p\right)+\alpha_{n} d\left(s_{n}^{\prime}, p\right) \\
& \leq\left(1-\alpha_{n}\right) \operatorname{dist}\left(s_{n}, p\right)+\alpha_{n} \operatorname{dist}\left(s_{n}^{\prime}, \mathcal{U} p\right) \\
& \leq\left(1-\alpha_{n}\right) H\left(\mathcal{U}_{n}, \mathcal{U} p\right)+\alpha_{n} H\left(\mathcal{U}_{n}, \mathcal{U} p\right)
\end{aligned}
$$

As $d(p, \mathcal{U} p)=0 \leq \frac{1}{2} d\left(x_{n}, p\right)$,

$$
\begin{aligned}
H\left(\mathcal{U} x_{n}, \mathfrak{U} p\right) & \leq \alpha \operatorname{dist}\left(p, \mathcal{U} x_{n}\right)+\beta \operatorname{dist}\left(x_{n}, \mathcal{U} p\right)+(1-\alpha-\beta) d\left(x_{n}, p\right) \\
& \leq \alpha\left\{\operatorname{dist}(p, \mathcal{U} p)+\operatorname{dist}\left(\mathfrak{U} p, \mathcal{U} x_{n}\right)\right\}+\beta\left\{d\left(x_{n}, p\right)+\operatorname{dist}(p, \mathfrak{U} p)\right\} \\
& +(1-\alpha-\beta) d\left(x_{n}, p\right) \\
& \leq \alpha H\left(\mathcal{U} x_{n}, \mathfrak{U} p\right)+(1-\alpha) d\left(x_{n}, p\right) \\
& \leq d\left(x_{n}, p\right) .
\end{aligned}
$$

Also,

$$
\begin{aligned}
H\left(U_{y_{n}}, \mathfrak{U} p\right) & \leq \alpha \operatorname{dist}\left(p, \mathfrak{U}_{n}\right)+\beta \operatorname{dist}\left(y_{n}, \mathfrak{U} p\right)+(1-\alpha-\beta) d\left(y_{n}, p\right) \\
& \leq \alpha\left\{\operatorname{dist}(p, \mathcal{U} p)+\operatorname{dist}\left(\mathfrak{U} p, \mathfrak{U}_{n}\right)\right\}+\beta\left\{d\left(y_{n}, p\right)+\operatorname{dist}(p, \mathcal{U} p)\right\}+ \\
& +(1-\alpha-\beta) d\left(y_{n}, p\right) \\
& \leq \alpha H\left(\mathfrak{U} y_{n}, \mathfrak{U} p\right)+(1-\alpha) d\left(y_{n}, p\right) \\
& \leq d\left(y_{n}, p\right)
\end{aligned}
$$


and

$$
\begin{aligned}
d\left(y_{n}, p\right) & =d\left(\left(1-\beta_{n}\right) x_{n} \oplus \beta_{n} s_{n}, p\right) \\
& \leq\left(1-\beta_{n}\right) d\left(x_{n}, p\right) \oplus \beta_{n} d\left(s_{n}, p\right) \\
& \leq\left(1-\beta_{n}\right) d\left(x_{n}, p\right)+\beta_{n} H\left(\mathcal{U} x_{n}, \mathcal{U} p\right) \\
& \leq\left(1-\beta_{n}\right) d\left(x_{n}, p\right)+\beta_{n} d\left(x_{n}, p\right) \\
& \leq d\left(x_{n}, p\right) .
\end{aligned}
$$

From (29) and (30) we obtain that

$$
H\left(\mathcal{U} y_{n}, \mathcal{U} p\right) \leq d\left(x_{n}, p\right) .
$$

Now using (30) and (31) in (27), we have

$$
d\left(x_{n+1}, p\right) \leq d\left(x_{n}, p\right) .
$$

Thus, for all $n \geq 1, d\left(x_{n+1}, p\right) \leq d\left(x_{n}, p\right)$ that is, $\left\{d\left(x_{n}, p\right)\right\}$ is decreasing and consequently, $\lim _{n \rightarrow \infty} d\left(x_{n}, p\right)$ exists.

(2) We now prove that $\lim _{n \rightarrow \infty} d\left(x_{n}, s_{n}\right)=0$.

From (1), we know that for each $p \in F(\mathcal{U}), \lim _{n \rightarrow \infty} d\left(x_{n}, p\right)$ exists. For some $r \geq 0$, let

$$
\lim _{n \rightarrow \infty} d\left(x_{n}, p\right)=r .
$$

From (28) and on taking the limit superior as $n \rightarrow \infty$, we have

$$
\limsup _{n \rightarrow \infty} d\left(s_{n}, p\right) \leq \limsup _{n \rightarrow \infty} H\left(\mathcal{U} x_{n}, \mathcal{U} p\right) \leq \limsup _{n \rightarrow \infty} d\left(x_{n}, p\right)=r,
$$

that is,

$$
\limsup _{n \rightarrow \infty} d\left(s_{n}, p\right) \leq r
$$

Similarly, from (28) and on taking limit superior as $n \rightarrow \infty$, we get

$$
\limsup _{n \rightarrow \infty} d\left(s_{n}^{\prime}, p\right) \leq r
$$

and hence

$$
r=\lim _{n \rightarrow \infty} d\left(x_{n+1}, p\right)=\lim _{n \rightarrow \infty} d\left(1-\alpha_{n} s_{n}+\alpha_{n} s_{n}^{\prime}, p\right) .
$$

Now, by applying Lemma 4, we get

$$
\limsup _{n \rightarrow \infty} d\left(s_{n}, s_{n}^{\prime}\right)=0 \text {. }
$$

From (30), we have

$$
d\left(y_{n}, p\right) \leq d\left(x_{n}, p\right) .
$$

On taking limit superior as $n \rightarrow \infty$, we obtain that

$$
\limsup _{n \rightarrow \infty} d\left(y_{n}, p\right) \leq r .
$$

Note that

$$
\begin{aligned}
d\left(x_{n+1}, p\right) & \leq\left(1-\alpha_{n}\right) d\left(s_{n}, p\right)+\alpha_{n} d\left(s_{n}^{\prime}, p\right) \\
& \leq d\left(s_{n}, p\right)-\alpha_{n} d\left(s_{n}, p\right)+\alpha_{n} d\left(s_{n}, p\right)+\alpha_{n} d\left(s_{n}, s_{n}^{\prime}\right) \\
& \leq d\left(s_{n}, p\right)+\alpha_{n} d\left(s_{n}, s_{n}^{\prime}\right) .
\end{aligned}
$$




$$
r \leq \liminf _{n \rightarrow \infty} d\left(s_{n}, p\right) .
$$

Combining (34) and (39), we have

$$
\limsup _{n \rightarrow \infty} d\left(s_{n}, p\right)=r
$$

Also,

$$
\begin{aligned}
d\left(s_{n}, p\right) & \leq d\left(s_{n}, s_{n}^{\prime}\right)+d\left(s_{n}^{\prime}, p\right) \\
& \leq d\left(s_{n}, s_{n}^{\prime}\right)+\operatorname{dist}\left(s_{n}^{\prime}, \mathcal{U} p\right) \\
& \leq d\left(s_{n}, s_{n}^{\prime}\right)+H\left(\mathcal{U} y_{n}, \mathcal{U} p\right) \\
& \leq d\left(s_{n}, s_{n}^{\prime}\right)+d\left(y_{n}, p\right) .
\end{aligned}
$$

By taking limit inferior as $n \rightarrow \infty$ on both sides, we have

$$
r \leq \liminf _{\rightarrow \infty} d\left(y_{n}, p\right) .
$$

Employing (34) and (40), yields

$$
\lim _{n \rightarrow \infty} d\left(y_{n}, p\right)=\lim _{n \rightarrow \infty} d\left(\left(1-\beta_{n} x_{n}+\beta_{n} s_{n}, p\right)=r .\right.
$$

From (32), (34), (40) and applying Lemma 5, we get

$$
\lim _{n \rightarrow \infty} d\left(x_{n}, s_{n}\right)=0 .
$$

Now, we present the existence result associated with multivalued generalized $(\alpha, \beta)$-nonexpansive mapping.

Theorem 2. Let $M$ and $U: M \longrightarrow \Omega(M)$ be as in Lemma 6. Fix $x_{1} \in M$ such that $x_{1} \preceq s_{1}$. If $\left\{x_{n}\right\}$ is a sequence given by (6) then the condition $\triangle-\lim _{n} x_{n}=x$ and $\lim _{n \rightarrow \infty} d\left(x_{n}, s_{n}\right)=0$ are satisfied then $x \in F(\mathcal{U})$.

Proof. Since $\triangle-\lim _{n} x_{n}=x$, Lemma 7 implies that $x_{n} \preceq x$ for all $n \geq 1$.

Utilizing the $(\alpha, \beta)-$ nonexpansiveness of $\mathcal{U}$ and $\lim _{n \rightarrow \infty} d\left(x_{n}, s_{n}\right)=0$, we have $z \in \mathcal{U} x$. Further,

$$
\begin{aligned}
d\left(z, x_{n}\right) & \leq d\left(z, s_{n}\right)+d\left(s_{n}, x_{n}\right) \\
\limsup _{n \rightarrow \infty} d\left(z, x_{n}\right) & \leq \limsup _{n \rightarrow \infty}\left[d\left(z, s_{n}\right)+d\left(s_{n}, x_{n}\right)\right] \\
& \leq \limsup _{n \rightarrow \infty} d\left(z, s_{n}\right) \\
& \leq \limsup _{n \rightarrow \infty} \operatorname{dist}\left(z, s_{n}\right) \\
& \leq \limsup _{n \rightarrow \infty} H\left(U x, U x_{n}\right) \\
& \leq \limsup _{n \rightarrow \infty} d\left(x, x_{n}\right) .
\end{aligned}
$$

Thus, from the uniqueness of the asymptotic center we have $z=x$ where $z \in \mathfrak{U} x$.

Here we discuss the convergence result regarding to our proposed mapping.

Theorem 3. Let $M$ and $\mathcal{U}: M \longrightarrow \Omega(M)$ be as in Lemma 6 with $F(\mathcal{U}) \neq \varnothing$. Fix $x_{1} \preceq s_{1} \in \mathcal{U} x_{1}$. If $\left\{x_{n}\right\}$ is a sequence defined by (6), then $\left\{x_{n}\right\} \triangle$-converges to an element of $F(\mathcal{U})$. 
Proof. It follows from Lemma 7 that $\lim _{n \rightarrow \infty} d\left(x_{n}, p\right)$ exists for each $p \in F(\mathcal{U})$. So, $\left\{x_{n}\right\}$ is bounded and $\lim _{n \rightarrow \infty} d\left(x_{n}, s_{n}\right)=0$, where $s_{n} \in \mathcal{U} x_{n}$.

Denote $\varrho_{l}\left(x_{n}\right)=\bigcup A_{k}\left(\left\{u_{n}\right\}\right)$ where the union is taken over all subsequence $\left\{u_{n}\right\}$ of $\left\{x_{n}\right\}$. We now prove that $\left\{x_{n}\right\}$ is $\triangle$-convergent to a fixed point of $\mathcal{U}$. First we show $\varrho_{l}\left(x_{n}\right) \subset F(\mathcal{U})$ and therefore assert that $\varrho_{l}\left(x_{n}\right)$ is singleton. To show $\varrho_{l}\left(x_{n}\right) \subset F(\mathcal{U})$. Let $y \in \varrho_{l}\left(x_{n}\right)$. So there exists a subsequence $\left\{y_{n}\right\}$ of $\left\{x_{n}\right\}$ such that $A\left(\left\{y_{n}\right\}\right)=\{y\}$. As a consequence of Lemma 3 and Lemma 4 , there exists a subsequence $\left\{t_{n}\right\}$ of $\left\{y_{n}\right\}$ so that $\triangle-\lim _{n} t_{n}=t$ and $t \in M$.

As $\lim _{n \rightarrow \infty} d\left(x_{n}, s_{n}\right)=0$ and $\left\{t_{n}\right\}$ is a subsequence of $\left\{x_{n}\right\}$, we have that $\lim _{n \rightarrow \infty} d\left(t_{n}, \mathcal{U} t_{n}\right)=0$. By Theorem 2, we have $t \in \mathcal{U} t$ and hence $t \in F(\mathcal{U})$. Now we assert that $t=y$. Indeed, $t \neq y$ leads to a contradiction as

$$
\begin{aligned}
\limsup _{n \rightarrow \infty} d\left(t_{n}, t\right) & <\limsup _{n \rightarrow \infty} d\left(t_{n}, y\right) \\
& \leq \limsup _{n \rightarrow \infty} d\left(y_{n}, y\right) \\
& <\limsup _{n \rightarrow \infty} d\left(y_{n}, t\right) \\
& =\limsup _{n \rightarrow \infty} d\left(x_{n}, t\right) \\
& =\limsup _{n \rightarrow \infty} d\left(t_{n}, t\right),
\end{aligned}
$$

and hence, $t=y \in F(\mathcal{U})$. To show that $\varrho_{l}\left(x_{n}\right)$ is singleton set, let $\left\{y_{n}\right\}$ be a subsequence of $\left\{x_{n}\right\}$. From Lemma 3 and 4 , there exists a subsequence $\left\{t_{n}\right\}$ of $\left\{y_{n}\right\}$ such that $\triangle-\lim _{n} t_{n}=t$. Let $A\left(\left\{y_{n}\right\}\right)=\{y\}$ and $A\left(\left\{x_{n}\right\}\right)=\{x\}$. As it is already proved that $t=y$ thus it is sufficient to demonstrate that $t=x$.

If $t \neq x$, then by Lemma $8,\left\{d\left(x_{n}, p\right)\right\}$ converges.

By uniqueness of asymptotic centers, we have

$$
\begin{aligned}
\limsup _{n \rightarrow \infty} d\left(t_{n}, t\right) & <\limsup _{n \rightarrow \infty} d\left(t_{n}, x\right) \\
& \leq \limsup _{n \rightarrow \infty} d\left(x_{n}, x\right) \\
& <\limsup _{n \rightarrow \infty} d\left(x_{n}, t\right) \\
& =\limsup _{n \rightarrow \infty} d\left(t_{n}, t\right),
\end{aligned}
$$

a contradiction that $t \neq x$, consequently $t=x \in F(\mathcal{U})$. Hence the conclusion follows.

In the following, we ascertain the strong convergence result which extends Theorem (1) in [39] for multivalued generalized $(\alpha, \beta)$-nonexpansive mapping in the setup of ordered CAT(0) space.

Theorem 4. Let $M$ and $\mathcal{U}: M \longrightarrow \Omega(M)$ be as in Lemma 6 such that $F(\mathcal{U}) \neq \varnothing$. Fix $x_{1} \in M$ such that $x_{1} \preceq s_{1} \in \mathcal{U} x_{1}$. If $\left\{x_{n}\right\}$ is a sequence described as (6) with $\sum_{n=1}^{\infty} \alpha_{n} \beta_{n}=\infty$, then $\left\{x_{n}\right\}$ converges to a fixed point of $U$ if and only if $\liminf _{n \rightarrow \infty} d\left(x_{n}, F(\mathcal{U})\right)=0$.

Proof. If the sequence $\left\{x_{n}\right\}$ converges to a fixed point $p \in F(\mathcal{U})$, then it is obvious that $\liminf _{n \rightarrow \infty} d\left(x_{n}, F(\mathcal{U})\right)=0$.

Conversely, suppose that $\liminf _{n \rightarrow \infty} d\left(x_{n}, F(\mathcal{U})\right)=0$. From Lemma 8, we have $\operatorname{dist}\left(x_{n+1}, p\right) \leq d\left(x_{n}, p\right)$ for any $p \in F(\mathcal{U})$. So, $\operatorname{dist}\left(x_{n+1}, F(\mathcal{U})\right) \leq d\left(x_{n}, F(\mathcal{U})\right.$ and hence $\left\{d\left(x_{n}, F(\mathcal{U})\right\}\right.$ forms a decreasing sequence that is bounded below by zero which implies that $\lim _{n \rightarrow \infty} d\left(x_{n}, F(\mathcal{U})\right)$ exists. 
To show $\left\{x_{n}\right\}$ is a Cauchy sequence in $M$, choose an arbitrary number, say, $\epsilon>0$. As $\liminf _{n \rightarrow \infty} d\left(x_{n}, F(\mathcal{U})\right)=0$ we have $\lim _{\rightarrow \infty} d\left(x_{n}, F(\mathcal{U})\right)=0$. Thus, there exists $n_{0}$ such that for all $n \geq n_{0}$, we have

$$
d\left(x_{n}, F(\mathcal{U})\right)<\frac{\epsilon}{4} .
$$

Specifically,

$$
\inf \left\{d\left(x_{n_{0}}, p\right): p \in F(\mathcal{U})\right\}<\frac{\epsilon}{4}
$$

Thus, there must exists $p \in F(\mathcal{U})$ such that $d\left(x_{n_{0}}, p\right)<\frac{\epsilon}{2}$. Now for $m, n \geq n_{0}$, we have

$$
\begin{aligned}
d\left(x_{m+n}, x_{n}\right) & \leq d\left(x_{m+n}, p\right)+d\left(p, x_{n}\right) \\
& <2 d\left(x_{n_{0}}, p\right)<2 \frac{\epsilon}{2}=\epsilon .
\end{aligned}
$$

Since $M \subset Y$ is closed, $\left\{x_{n}\right\}$ is a Cauchy sequence and consequently, converges in $M$. Let $\liminf _{n \rightarrow \infty} x_{n}=g$. Note that

$$
\begin{aligned}
\operatorname{dist}(g, \mathcal{U} g) & \leq \operatorname{dist}\left(g, x_{n}\right)+\operatorname{dist}\left(x_{n}, \mathfrak{U} x_{n}\right)+\operatorname{dist}\left(\mathcal{U} x_{n}, g\right) \\
& \leq d\left(x_{n}, g\right)+d\left(x_{n}, s_{n}\right)+H\left(\mathcal{U} x_{n}, \mathfrak{U} g\right) \\
& \leq d\left(x_{n}, g\right)+d\left(x_{n}, s_{n}\right)+H\left(\mathcal{U} x_{n}, \mathfrak{U} g\right) .
\end{aligned}
$$

On taking limit as $n \rightarrow \infty$, we have $g \in U$ g. This completes the proof.

\section{Remark 3.}

(1) For $\alpha=\beta=0$, our theorems extend the results in [27] to CAT(0) spaces.

(2) For $\alpha=\beta$, these results extend the results in [31,40] to CAT(0) spaces.

(3) Our results extend and improve results in [20] for monotone nonexpansive mapping in a CAT $(0)$ spaces.

\section{Numerical Experiments}

We start with the following example.

Example 2. Let $M=[0,2]$ and $Y$ a $C A T(0)$ space equipped with the order " $\geq$ " and the standard metric given by $d(v, y)=|v-y|$. Define $\mathcal{U}: M \longrightarrow \Omega(M)$ by

$$
u(v)=\left\{\begin{array}{l}
{\left[0, \frac{v}{2}\right] \quad \text { if } \quad 0 \leq v<\frac{1}{2}} \\
\{0\} \quad \text { if } \quad \frac{1}{2} \leq v \leq 1
\end{array}\right.
$$

Clearly, $u$ is monotone. Indeed, if $v \geq y$ for $v \in\left[\frac{1}{2}, 1\right]$ and $y \in\left[0, \frac{1}{2}\right)$, then for any $u_{v} \in \mathcal{U v}_{v}=\{0\}$, there exists $u_{y}=0 \in \mathcal{U} y$ such that $u_{v} \geq u_{y}$.

Note that $U$ does not satisfy the Condition $(C)$ and hence is not a nonexpansive multivalued mapping. If we take $v=\frac{1}{2}$ and $y=\frac{11}{20}$, then

$$
\begin{aligned}
\frac{1}{2} \operatorname{dist}(v, \mathcal{U v}) & \leq \frac{1}{2} d\left(\frac{1}{4}, \mathcal{U}\left(\frac{1}{4}\right)\right)=0 \\
\text { and } d(v, y) & =\left|\frac{1}{4}-\frac{11}{20}\right|=0.05>0 \\
\text { implies that } \frac{1}{2} \operatorname{dist}(v, \mathcal{U} v) & \leq d(v, y) . \\
H(\mathcal{U}, \mathcal{U} y) & =H\left(\mathcal{U}\left(\frac{1}{4}\right), \mathcal{U}\left(\frac{11}{20}\right)\right)
\end{aligned}
$$

Note that

$$
H\left(\mathcal{V}, U_{y}\right)=H\left(\mathcal{U}\left(\frac{1}{4}\right), \mathcal{U}\left(\frac{11}{20}\right)\right)=H\left(\left[0, \frac{1}{4}\right],\{0\}\right)=\frac{1}{4}>0.05 .
$$


Now, to demonstrate $U$ is a multivalued generalized $(\alpha, \beta)$-nonexpansive mapping, the following cases are considered:

Case 1: If $v, y \in\left[0, \frac{1}{2}\right)$, then

$$
H(\mathcal{U}, \mathcal{U} y)=\left|\frac{v-y}{2}\right| \leq \frac{1}{3}\left|v-\frac{y}{2}\right|+\frac{1}{6}\left|y-\frac{v}{2}\right|+\frac{1}{2}|v-y| .
$$

Case 2: The case $v, y \in\left(\frac{1}{2}, 1\right]$ is trivial.

Case 3: If $v \in\left[0, \frac{1}{2}\right]$ and $y \in\left(\frac{1}{2}, 1\right]$,

$$
\begin{aligned}
\alpha \operatorname{dist}\left(y, \mathcal{U v}_{v}\right)+\beta \operatorname{dist}(v, \mathcal{U} y) & +(1-\alpha-\beta) d(v, y) \\
& =\frac{1}{3}|v|+\frac{1}{6}\left|y-\frac{v}{2}+3 v-y\right| \\
& \geq \frac{1}{3}|v|+\frac{5}{12}|v| \geq \frac{3}{4}|v|>\frac{1}{2}|v|=H(\mathcal{U}, \mathcal{U} y) .
\end{aligned}
$$

Hence, $\mathfrak{U}$ is $\left(\frac{1}{3}, \frac{1}{6}\right)$ - nonexpansive multivalued mapping.

Now, we conduct some experiments to determine and compare the convergence behavior of iteration (6). In Table 1 we test the convergence of algorithms for different initial points. Fix parameters are $\alpha_{n}=\frac{1}{\sqrt{2 n+1}}, \beta_{n}=\frac{1}{\sqrt{n+1}}$ and choose the stopping criteria to be $\left\|x_{n}-x *\right\|<10^{-5}$ where $x^{*}$ is the solution of the fixed point problem. It is evident that S-iteration (6) converges faster to the fixed point of the multivalued generalized $(\alpha, \beta)$-nonexpansive mapping.

Table 1. Convergence comparison of Iteration Processes for different choices of initial points.

\begin{tabular}{cccc}
\hline Initial Points & \multicolumn{3}{c}{ Iteration Processes } \\
\hline & Mann & Ishikawa & S-iteration \\
0.1 & 183 & 79 & 13 \\
0.4 & 239 & 104 & 14 \\
0.6 & 234 & 108 & 14 \\
0.7 & 241 & 111 & 14 \\
\hline
\end{tabular}

In the figures, Figures 1 and 2 we test the convergence of different iterative processes for different choices of parameters. For this, we chose initial point $x_{1}=0.4$. Observe that for different choices of parameters the modified $S$-iteration (6) converges faster to the fixed point of the multivalued generalized $(\alpha, \beta)$-nonexpansive mapping.
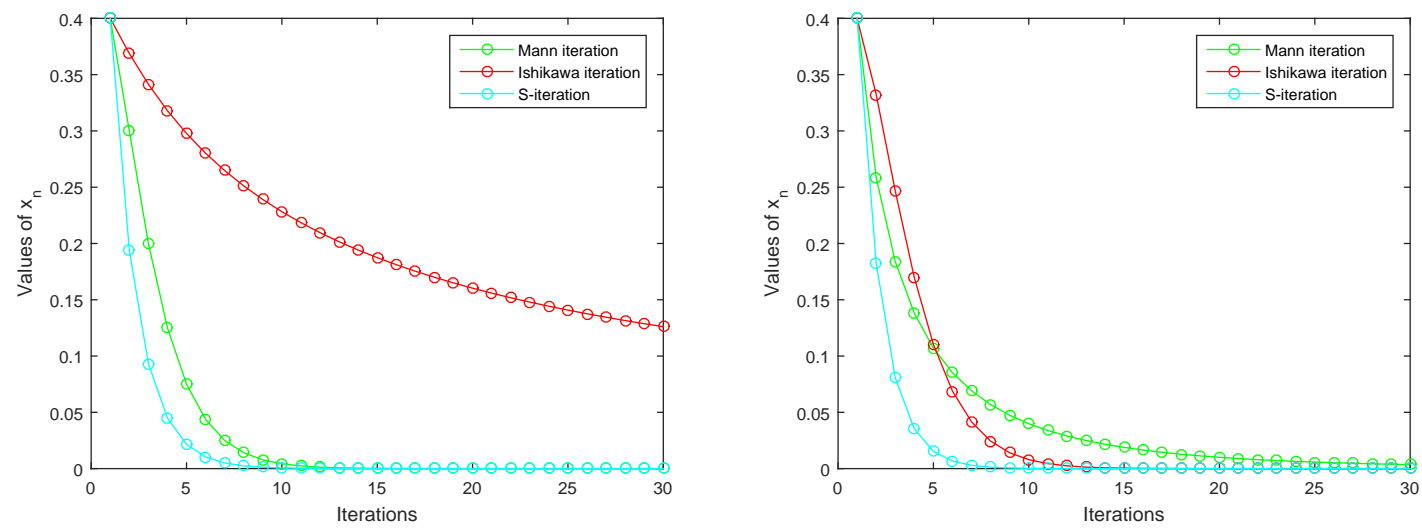

Figure 1. Comparison of Iteration Processes for different choices of parameters: $\alpha_{n}=\frac{n}{n+1}, \beta_{n}=\frac{1}{n+7}$ (left plot), $\alpha_{n}=\frac{1}{\sqrt{n+1}}$, $\beta_{n}=\frac{n}{n+3}$ (right plot). 

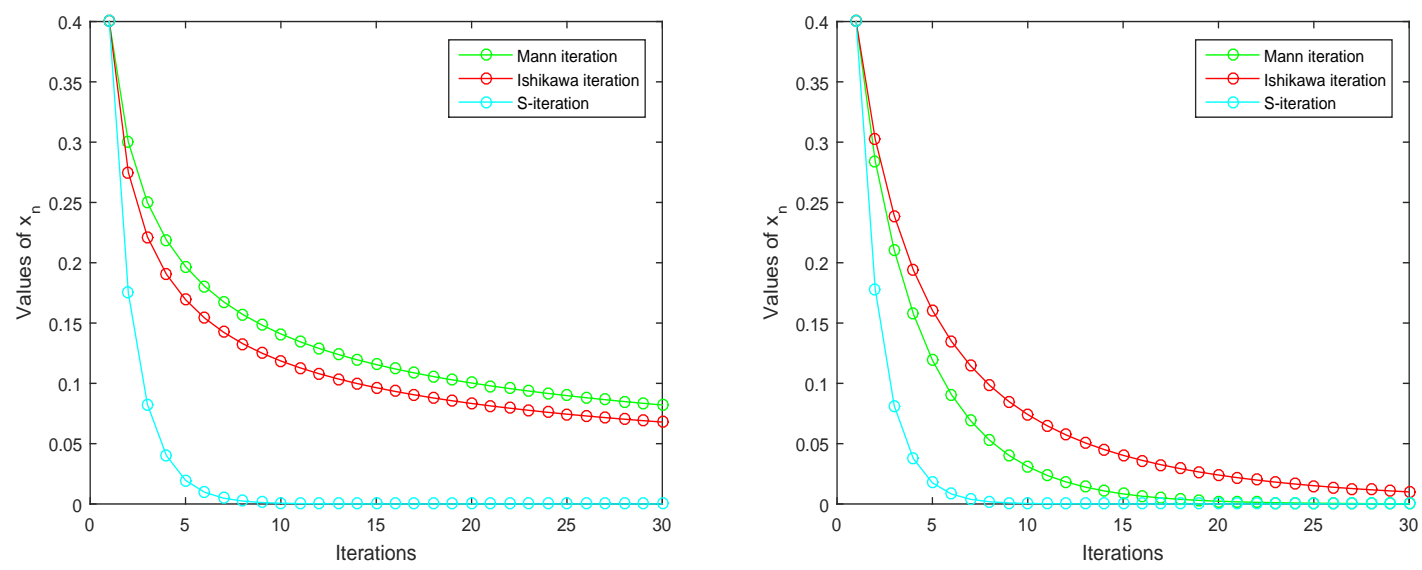

Figure 2. Comparison of Iteration Processes for different choices of parameters: $\alpha_{n}=\frac{1}{n+1}, \beta_{n}=\frac{1}{n+1}$ (left plot), $\alpha_{n}=\sqrt{\frac{n+1}{5 n+1}}$, $\beta_{n}=\frac{1}{\sqrt{2 n+5}}$ (right plot).

\section{Application to Integral Equations}

In this section, we show that our iterative scheme defined as in (6) can be used to find a solution of an integral equation. Let $Y=L^{2}([0,1], \mathbb{R})$ be the space of real valued functions on $[0,1]$ such that $\int_{-\infty}^{+\infty}|f(x)|^{2}<+\infty$. Since all Hilbert spaces are CAT(0) spaces then $L^{2}([0,1], \mathbb{R})$ is $\operatorname{CAT}(0)$. Define the order on $Y$ by

$$
y \preccurlyeq z \text { if and only if } y(t) \leq z(t)
$$

for all $t \in[0,1]$. Consider the following integral equation:

$$
y(t)=g(t)+\int_{0}^{1} F(t, s, y(s)) d s
$$

where $t \in[0,1], g \in L^{2}([0,1], \mathbb{R})$ and $F:[0,1] \times[0,1] \times L^{2}([0,1], \mathbb{R}) \rightarrow \mathbb{R}$. In addition, $F$ is measurable and satisfies the inequality:

$$
0 \leq|F(t, s, y)-F(t, s, z)| \leq|y-z|
$$

for $t, s \in[0,1]$ and $y, z \in L^{2}([0,1], \mathbb{R})$ such that $y \leq z$.

Suppose that there exists a nonnegative function $f(.,.) \in L^{2}([0,1] \times[0,1])$ and $Q<\frac{1}{2}$ satisfying

$$
|F(t, s, y)| \leq f(t, s)+Q|y|
$$

for $t, s \in[0,1]$ and $y \in L^{2}([0,1], \mathbb{R})$. Consider a closed ball $M$ of $L^{2}([0,1], \mathbb{R})$ centered at 0 with radius $\sigma$, i.e.,

$$
M=\left\{y \in L^{2}([0,1], \mathbb{R}) \quad \text { such that } \quad\|y\|_{L^{2}([0,1], \mathbb{R})} \leq \sigma\right\},
$$

where $\sigma$ is sufficiently large.

Define the operator $\mathcal{U}: L^{2}([0,1], \mathbb{R}) \rightarrow L^{2}([0,1], \mathbb{R})$ by

$$
u(y(t))=g(t)+\int_{0}^{1} F(t, s, y(s)) d s .
$$

Then $U(M) \subset M$. Clearly, $\mathcal{U}$ monotone nonexpansive mapping and hence monotone generalized $(\alpha, \beta)$-nonexpansive. Choose $Y=L^{2}([0,1], \mathbb{R})$ and $\mathcal{U}$ as in (44) in Theorem 3 , we get the following result. 
Theorem 5. Assuming the above stated assumptions, the sequence constructed by the iteration scheme (6) converges to a solution of the integral Equation (43).

\section{Conclusions}

In this paper, we presented a new type of mappings, monotone multivalued generalized $(\alpha, \beta)$-nonexpansive mappings. We have investigated the iterative approximation of the fixed points for such mappings in an ordered CAT(0) space utilizing the $S$-iteration algorithm and some convergence results were established. Through numerical experiments we have shown that the process (6) converges faster than all the leading schemes. The proposed mapping and results generalize and extend various results in CAT(0) space including $[20,27,31,40]$. Furthermore, we have also presented an application of the results in approximating the solution of integral equations.

Author Contributions: Conceptualization, M.A. and H.I.; methodology, H.I. and K.A.; validation, M.A., H.I. and M.D.1.S.; formal analysis, H.I. and K.A.; investigation, H.I. and K.A.; writing—original draft preparation, K.A.; writing—review and editing, M.A. and H.I.; supervision, M.A. and M.D.l.S.; project administration, M.D.1.S.; funding acquisition, M.D.1.S. All authors have read and agreed to the published version of the manuscript.

Funding: The authors are grateful to the Spanish Government for its support through grant RTI2018094336-B-100 (MCIU/AEI/FEDER, UE) and to the Basque Government for its support through Grant IT1207-19. The first author was supported by the Higher Education Commission of Pakistan (project No. 9340).

Institutional Review Board Statement: Not applicable.

Informed Consent Statement: Not applicable.

Data Availability Statement: Not applicable.

Acknowledgments: Authors are thankful to the reviewers and editor for their constructive comments which helped us a lot in improving the presentation of the paper.

Conflicts of Interest: The authors declare no conflict of interest.

\section{References}

1. Agarwal, R.P.; O’Regan, D.; Sahu, D.R. Fixed Point Theory for Lipschitzian-Type Mappings with Applications; Springer: New York, NY, USA, 2009; Volume 6.

2. Bridson, M.R.; Haefliger, A. Metric Spaces of Non-Positive Curvature; Springer Science \& Business Media: Berlin, Germany, 2013; Volume 319.

3. Niculescu, C.P.; Rovenţa, I. Fan's inequality in geodesic spaces. Appl. Math. Lett. 2009, 22, 1529-1533. [CrossRef]

4. Shabanian, S.; Vaezpour, S.M. A minimax inequality and its applications to fixed point theorems in cat (0) spaces. Fixed Point Theory Appl. 2011, 2011, 61. [CrossRef]

5. Haeiger, A.; Bridson, M.R. Metric Spaces of Non-Positive Curvature, Fundamental Principle of Mathematical Sciences; Springer: Berlin, Germany, 1999; Volume 319.

6. Kirk, W.A. Fixed point theorems in CAT(0) spaces and R-trees. Fixed Point Theory Appl. 2004, 2004, 309-316. [CrossRef]

7. Shafrir, I.; Reich, S. Nonexpansive iterations in hyperbolic spaces. Nonlinear Anal. 1990, 15, 537.

8. Brown, K.S. Buildings; Springer: New York, NY, USA, 1998; Volume 319.

9. Markin, J.T. A fixed point theorem for set valued mappings. Bull. Am. Math. Soc. 1968, 74, 639-640. [CrossRef]

10. Nadler, S.B., Jr. Multi-valued contraction mappings. Pac. J. Math. 1969, 30, 475-488. [CrossRef]

11. Altun, I.; Simsek, H. Some fixed point theorems on dualistic partial metric spaces. J. Adv. Math. Stud. 2008, 1, 1-9.

12. Mlaiki, N.; Abodayeh, K.; Aydi, H.; Abdeljawad, T.; Abuloha, M. Rectangular metric-like type spaces and related fixed points. J. Math. 2018, 2018, 3581768. [CrossRef]

13. Oltra, S.; Valero, O. Banach's Fixed Point Theorem for Partial Metric Spaces; Università degli Studi di Trieste. Dipartimento di Matematica e Informatica: Trieste, Italy, 2004.

14. Shukla, S. Partial b-metric spaces and fixed point theorems. Mediter. J. Math. 2014, 11, 703-711.

15. Aydi, H.; Abbas, M.; Vetro, C. Partial hausdorff metric and nadler's fixed point theorem on partial metric spaces. Topol. Appl. 2012, 159, 3234-3242. [CrossRef]

16. Lim, T. A fixed point theorem for multivalued nonexpansive mappings in a uniformly convex banach space. Bull. Am. Math. Soc. 1974, 80, 1123-1126. [CrossRef] 
17. Shimizu, T.; Takahashi, W. Fixed points of multivalued mappings in certain convex metric spaces. Topol. Methods Nonlinear Anal. 1996, 8, 197-203. [CrossRef]

18. Dhompongsa, S.; Kaewkhao, A.; Panyanak, B. Lim's theorems for multivalued mappings in cat (0) spaces. J. Math. Anal. Appl. 2005, 312, 478-487. [CrossRef]

19. Razani, A.; Shabani, S. Approximating fixed points for nonself mappings in cat (0) spaces. Fixed Point Theory Appl. 2011, $2011,65$. [CrossRef]

20. Uddin, I.; Nieto, J.J.; Ali, J. One-step iteration scheme for multivalued nonexpansive mappings in CAT (0) spaces. Mediter. J. Math. 2016, 13, 1211-1225. [CrossRef]

21. Lu, H.; Lan, D.; Hu, Q.; Yuan, G. Fixed point theorems in CAT(0) spaces with applications. J. Inequal. Appl. 2014, $2014,320$. [CrossRef]

22. Bartolini, M.P.I.; Ciaccia, P. String Matching with Trees Using an Approximate Distance. In Spir Lecture Notes in Computer Science; Springer: Berlin/Heidelberg, Germany, 2002. [CrossRef]

23. Kirk, W.A.; Espinola, R. Fixed point theorems in r-trees with applications to graph theory. Topol. Appl. 2006, 153, $1046-1055$.

24. La Sen, M.D. About fixed points in CAT(0) spaces under a combined structure of two self-mappings. J. Math. 2017, 2017, 13. [CrossRef]

25. Uddin, I.; Ali, J.; Nieto, J.J. An iteration scheme for a family of multivalued mappings in CAT (0) spaces with an application to image recovery. Rev. Real Acad. Cienc. Exactas Físicas Nat. Ser. A Matemáticas 2018, 112, 373-384. [CrossRef]

26. Suzuki, T. Fixed point theorems and convergence theorems for some generalized nonexpansive mappings. J. Math. Anal. Appl. 2008, 340, 1088-1095. [CrossRef]

27. Abkar, A.; Eslamian, M. Fixed point theorems for suzuki generalized nonexpansive multivalued mappings in banach spaces. Fixed Point Theory Appl. 2010, 2010, 457935. [CrossRef]

28. Chang, S.; Wen, C.; Yao, J. Proximal point algorithms involving cesaro type mean of asymptotically nonexpansive mappings in cat (0) spaces. J. Nonlinear Sci. Appl. 2016, 9, 4317-4328. [CrossRef]

29. Khan, S.H.; Abbas, M. Common fixed points of two multivalued nonexpansive maps in Kohlenbach hyperbolic spaces. Fixed Point Theory Appl. 2014, 2014, 181. [CrossRef]

30. Aoyama, K.; Kohsaka, F. Fixed point theorem for $\alpha$-nonexpansive mappings in Banach spaces. Nonlinear Anal. Theory Methods Appl. 2011, 74, 4387-4391. [CrossRef]

31. Iqbal, H.; Abbas, M.; Husnine, S.M. Existence and approximation of fixed points of multivalued generalized $\alpha$-nonexpansive mappings in banach spaces. Numer. Algor. 2020, 85, 1029-1049. [CrossRef]

32. Amini-Harandi, A.; Fakhar, M.; Hajisharifi, H.R. Approximate fixed points of $\alpha$-nonexpansive mappings. J. Math. Anal. Appl. 2018, 467, 1168-1173. [CrossRef]

33. Laowang, W.; Panyanak, B. Strong and convergence theorems for multivalued mappings in CAT(0) spaces. J. Inequal. Appl. 2009, 2009, 730132. [CrossRef]

34. Panyanak, B. Mann and Ishikawa iterative processes for multivalued mappings in Banach spaces. Comput. Math. Appl. 2007, 54, 872-877. [CrossRef]

35. Dhompongsa, S.; Panyanak, B. On $\triangle$-convergence theorems in CAT (0) spaces. Comput. Math. Appl. 2008, 56, 2572-2579. [CrossRef]

36. Laowang, W.; Panyanak, B. Approximating fixed points of nonexpansive nonself mappings in cat (0) spaces. Fixed Point Theory Appl. 2009, 2010, 367274. [CrossRef]

37. Agarwal, R.P.; Regan, D.O.; Sahu, D.R. Iterative construction of fixed points of nearly asymptotically nonexpansive mappings. J. Nonlinear Convex Anal. 2007, 8, 61-79.

38. Pandey, R.; Pant, R.; Al-Rawashdeh, A. Fixed point results for a class of monotone nonexpansive type mappings in hyperbolic spaces. J. Funct. Spaces 2018, 2018, 5850181. [CrossRef]

39. Khan, S.H. Convergence of iterates with errors of asymptotically quasi-nonexpansive mappings and applications. J. Math. Anal. Appl. 2007, 328, 821-829.

40. Pant, R.; Shukla, R. Approximating fixed points of generalized $\alpha$-nonexpansive mappings in banach spaces. Numer. Funct. Anal. Optimiz. 2017, 38, 248-266. [CrossRef] 